University of Nebraska - Lincoln

DigitalCommons@University of Nebraska - Lincoln

Faculty Publications in Computer \& Electronics Electrical \& Computer Engineering, Department Engineering (to 2015)

1998

\title{
Error Performance of the 13-Channel DS CDMA WATM LAN
}

\author{
Beata J. Wysocki \\ University of Nebraska-Lincoln \\ Tadeusz Wysocki \\ University of Nebraska-Lincoln, wysocki@uow.edu.au \\ Hans J. Zepernick \\ Curtin University of Technology
}

Follow this and additional works at: https://digitalcommons.unl.edu/computerelectronicfacpub

Part of the Computer Engineering Commons

Wysocki, Beata J.; Wysocki, Tadeusz; and Zepernick, Hans J., "Error Performance of the 13-Channel DS CDMA WATM LAN" (1998). Faculty Publications in Computer \& Electronics Engineering (to 2015). 29. https://digitalcommons.unl.edu/computerelectronicfacpub/29

This Article is brought to you for free and open access by the Electrical \& Computer Engineering, Department of at DigitalCommons@University of Nebraska - Lincoln. It has been accepted for inclusion in Faculty Publications in Computer \& Electronics Engineering (to 2015) by an authorized administrator of DigitalCommons@University of Nebraska - Lincoln. 


\title{
Error Performance of the 13-Channel DS CDMA WATM LAN
}

\author{
Beata J. Wysocki, Tadeusz A. Wysocki, Hans J. Zepernick \\ Cooperative Research Centre - Broadband Telecommunications and Networking \\ Curtin University of Technology \\ GPO Box U1987, Perth WA6845, Australia \\ email: tad@atri.curtin.edu.au \\ tel: +61892667892 \\ fax: +61892663244
}

\begin{abstract}
In the paper, we present simulation results for the 13 channel DS CDMA WATM LAN utilising optimized complex spreading signatures based on Walsh-Rademacher functions. The method to obtain those optimized spreading signatures, as well as the full set of the coefficients giving the minimum level of cross-correlation between any pair of the channels is shown. The resultant system BER and the distribution of errors within encapsulated WATM cells is given. The obtained results indicate that with the application of a hybrid ARQ scheme, capable of correcting 10 errors, the number of WATM cells which would require retransmission is in the order of $1-2 \%$.
\end{abstract}

\section{INTRODUCTION}

ATM (Asynchronous Transfer Mode) is the commonly accepted standard for the broadband networks, and is also becoming accepted as a common nominator for all types of services and networks. Also, there has been widespread use of wireless communications to support users' requirements for wireless access and terminal mobility in such scenarios as cellular telephony networks and narrowband wireless LANs. As a further advancement, users are now beginning to require broadband services and terminal mobility to be provided together through wireless access to ATM networks.

A major challenge in the design of WATM networks is the need to overcome the severe degradation of channel bit error rate resulting from impairments to the wireless channel such as those caused by multi-path propagation [1]. Spread spectrum techniques, such as direct sequence code division multiple access (CDMA), can be effective in combating multi-path effects, while also providing good tolerance to interference from other devices operating in the same frequency band. However, only a small processing gain is achievable due to the total available bandwidth of tens of $\mathrm{MHz}$ and the high data rate of several Mbps. Consequently, in-band jammers, like other channels of the same WLAN acquired by means of code division multiple access (CDMA) cause severe multiple access interference (MAI), which may block the communication.
In theory, cancellation of that type of interference is possible if each of the users utilise orthogonal signals to transmit the data [2]. If the delays between transmitters and receiver are anyhow different, as is generally the case of terminal to base station (BS) transmission, the signals received by the $B S$ cannot be regarded as orthogonal. Even within a $50 \mathrm{~m}$ coverage area those differences may be in the order of a few spreading code symbols (chips) depending on the data rate. This effect is particularly critical for very short spreading sequences, like 16-bits Walsh-Rademacher functions.

In [3], we have proposed the method to reduce the ISI and MAI for DS CDMA wireless networks, and in this paper, we consider application of this method to derive the spreading signatures based on the 16-bits WalshRademacher functions [4] for the DS CDMA ATM WLAN.

The paper is organized as follows. In Section II, we briefly discuss the method of designing the complex multilevel spreading signatures based on the known set of binary codes. Section III deals with the practical implementation of the design method, and optimization of parameters for the set of complex signatures. In Section IV, we present simulation results for the 13 channel ATM WLAN utilizing these signatures, and Section V concludes the paper.

\section{DESIGN METHOD}

In [3], we have described the method to reduce ISI and MAI for non-synchronized CDMA signals by means of a modification to the carrier waveform. The modified carrier has been obtained by a regular distortion to the frequency of the original carrier, resulting in the ith user line signal $s_{i}(t)$ expressed by:

$$
s_{i}(t)=g_{i}(t) \cdot \cos \left[\omega_{c} t+\int_{0}^{t} w_{i}(\tau) d \tau+\phi_{i}(t)\right],
$$

where $w_{i}(t)$ - frequency distorting function which can be optimized to achieve minimum value of cross-correlation among all users, and to minimize the off-peak auto- 
correlation of the ith line signal, $\phi_{i}(t)$ - information carrying phase component.

To simplify simulation process, we have applied here a base-band equivalent of the method. If follows from the fact that for the real spreading code $g_{i}(t)$, equation (1) can be rewritten in the exponential form as:

$$
s_{i}(t)=g_{i}(t) \cdot \operatorname{Re}\left\{e^{j \omega_{c} t} \exp \left[j \int_{0}^{t} w_{i}(\tau) d \tau\right] e^{\phi_{i}(t)}\right\} .
$$

The complex envelope [2] $\sigma(t)$ of such a signal is given by:

$$
\sigma(t)=g_{i}(t) \exp \left[j \int_{0}^{t} w_{i}(\tau) d \tau\right] e^{\phi_{i}(t)}=\tilde{g}_{i}(t) e^{\phi_{i}(t)},
$$

Hence, the function

$$
\tilde{g}_{i}(t)=g_{i}(t) \exp \left[j \int_{0}^{t} w_{i}(\tau) d \tau\right]
$$

can be regarded as an analogue spreading waveform, leading, without any changes to the cosinusoidal carrier, to the same line signal $s_{i}(t)$, as the original binary spreading code $g_{i}(t)$ together with the previously described modification to the carrier waveform.

Because signal is usually processed using digital signal processing (DSP) technology in the receiver, instead of the analogue spreading waveform $\tilde{g}_{i}(t)$, one can use a multilevel complex spreading signature $\hat{g}_{i}(t)$. The length of the signature $\hat{g}_{i}(t)$ equals to the length of the original signature $g_{i}(t)$ multiplied by the number of samples per chip used in the receiver, and its pulse levels are weighted by the factor

$$
W_{i}(t)=\exp \left[j \int_{0}^{t} w_{i}(\tau) d \tau\right]
$$

Such an approach allows for the baseband correlational detection of the signals, easier optimization of the functions $w_{i}(t)$ for the given set of binary signatures, and facilitates implementation of the receivers.

In order to optimize the spreading sequences for the use in a 13 channel DS CDMA ATM WLAN, we have chosen a subset of 13 orthogonal Walsh-Rademacher functions [2] as a set of binary spreading codes $g_{i}(t)$, $i=1,2, \ldots, 13$. These functions are listed in the Table 1 .

To minimize the cross-correlation between any pair of the spreading signatures, independently of the relative delay (for a delay different than zero the sequences are, in general, not orthogonal), we applied the described method with the functions $w_{i}(t), i=1,2, \ldots, 13$, being of the form:

$$
w_{i}(t)=2 \pi\left[\alpha_{i} \zeta(t / 16)+\beta_{i} \zeta(t / 8)+\gamma_{i} \zeta(t / 4)\right],
$$

where $\zeta(t)$ - the triangular wave defined as:

$$
\zeta(t)=\sum_{n=-\infty}^{\infty} \lambda(t-n)
$$

\begin{tabular}{|c|c|}
\hline No. & Binary spreading sequence \\
\hline 1 & $1111-1-1-1-111111-1-1-1-1$ \\
\hline 2 & $11-1-1-1-111111-1-1-1-111$ \\
\hline 3 & $-1-11111-1-111-1-1-1-111$ \\
\hline 4 & $-1-1111-1-111111-1-111-1-1$ \\
\hline 5 & $11-1-1111-1-1111-1-1111-1-1$ \\
\hline 6 & $1-1-1111-1-1111-1-1.11-1-11$ \\
\hline 7 & $-111-1-111-111-1-1111-1-11$ \\
\hline 8 & $-111-11-1-1111-1-111-1111-1$ \\
\hline 9 & $1-1-111-1111-111-1-111-1111-1$ \\
\hline 10 & $1-11-1-11-111-1-1-1-11-11$ \\
\hline 11 & $-11-111-11-11-11-1-11-11$ \\
\hline 12 & 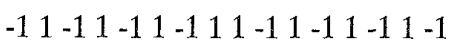 \\
\hline 13 & 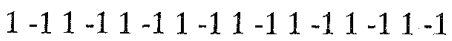 \\
\hline
\end{tabular}

and

$$
\lambda(t)=\left\{\begin{array}{rc}
0, & t<0 \\
4 t-1, & 0 \leq t<0.5 \\
-4 t+3, & 0.5 \leq t<1 \\
0, & t \geq 1
\end{array} .\right.
$$

Table 1: Set of 13 spreading sequences.

\section{OPTIMIZATION OF THE SIGNATURE SET}

Optimization problem in the considered case can be formulated as follows:

- find the values of the coefficients $\alpha_{i}, \beta_{i}$, and $\gamma_{i}$; $i=1,2, \ldots, 13$, minimizing the maximum value $\kappa(A)$, 


$$
A=\left[\begin{array}{lll}
\alpha_{1} & \ldots & \alpha_{13} \\
\beta_{1} & \ldots & \beta_{13} \\
\gamma_{1} & \ldots & \gamma_{13}
\end{array}\right]
$$

of the cross-correlation coefficients between any pair of the spreading codes, independently of the relative delay between them.

Due to the bandwidth limitations, we want to keep the magnitudes of these coefficients as low as possible. Therefore, one needs to impose constraints on the values of these coefficients. In our case, we required them to fulfil the following:

$$
\forall(i=1,2, \ldots, 13)\left\{\begin{aligned}
\left|\alpha_{i}\right| & \leq 5 \\
\left|\beta_{i}\right| & \leq 5 \\
\left|\gamma_{i}\right| & \leq 5 \\
\left|\alpha_{i}\right|+\left|\beta_{i}\right| & +\left|\gamma_{i}\right| \leq 10
\end{aligned}\right.
$$

Since the function to be minimized is highly irregular having many local maxima and minima, we have used the following algorithm to find the solution:

i. In the first step of the optimization we set:

$$
\left\{\begin{array}{l}
\alpha_{i}=\alpha, i=1,2, \ldots, 13 \\
\beta_{i}=\beta, i=1,2, \ldots, 13 \\
\gamma_{i}=\gamma, i=1,2, \ldots, 13
\end{array}\right.
$$

and calculated the values of $\kappa(A)$ for the area satisfying the condition (10), with grid step being equal to 0.05 for each of the parameters $\alpha, \beta$, and $\gamma$.

ii. Next we perform optimization for all 39 variables using the Nelder-Meade simplex search [5] implemented in MATLAB as the function fmins [6]. For the starting point, we choose

$$
A^{*}=\left[\begin{array}{c}
\alpha^{*} \\
\beta^{*} \\
\gamma^{*}
\end{array}\right] \otimes\left[\begin{array}{llllllllllll}
1 & 1 & 1 & 1 & 1 & 1 & 1 & 1 & 1 & 1 & 1 & 1
\end{array}\right],
$$

where: $\otimes$ denotes the Kronecker product, $\alpha^{*}, \beta^{*}$, and $\gamma$ are the values of $\alpha, \beta$, and $\gamma$, respectively, for which the function $\kappa(A)$ reaches the minimum in the previous step.

The optimized values of the coefficients $\alpha, \beta$, and $\gamma$ are given in the Table 2.

For this set of coefficients, the function $\kappa\left(A_{\text {opt }}\right)=\kappa_{\text {min }}=0.2733$.

Since we can regard this results as a local minimum only, we have also tried another optimization approach. The starting points for the MATLAB function fmins have been chosen randomly from the whole considered domain. However, even though the procedure have been repeated 10000 times, no better solution has been found.

Table 2: Set of the optimized coefficients.

\begin{tabular}{|c|c|c|c|}
\hline No. & $\alpha$ & $\beta$ & $\gamma$ \\
\hline 1 & -4.2315 & -2.1554 & -2.8487 \\
\hline 2 & -4.2040 & -2.0251 & -2.7369 \\
\hline 3 & -4.2127 & -2.0521 & -2.7685 \\
\hline 4 & -4.2597 & -2.0233 & -2.8507 \\
\hline 5 & -4.1610 & -2.1498 & -2.8281 \\
\hline 6 & -4.1504 & -2.1694 & -2.8278 \\
\hline 7 & -4.2512 & -2.1321 & -2.7522 \\
\hline 8 & -4.3205 & -2.0186 & -2.6968 \\
\hline 9 & -4.2259 & -2.0962 & -2.7669 \\
\hline 10 & -4.2763 & -2.0668 & -2.7818 \\
\hline 11 & -4.2678 & -2.1340 & -2.9848 \\
\hline 12 & -4.1794 & -2.1598 & -2.8193 \\
\hline 13 & -4.2054 & -2.0276 & -2.7752 \\
\hline
\end{tabular}

Therefore, we have decided to use the solution given in Table 2 in the simulation of the system.

\section{SIMULATION OF DS CDMA WATM LAN}

In order to simulate the 13 channel DS CDMA WATM LAN we assumed the following:

- an encapsulated WATM cell consisted of 524 bits (424 bits of an ATM cell plus 100 bits of an overhead),

- BPSK was used as a modulation technique,

- the contents of each WATM cell was random,

- relative delay between the signals corresponding to different channels was random with the delay step of 0.25 of a chip duration,

- the received power was equal for all of the channels.

The receiver antenna was simulated as a sum of the signals arriving from different channels, and the detection was a correlational one. The simulation was repeated for 500 cells for each of the channels.

The obtained results show that the quality of transmission depends slightly on the signature used to 
spread the signal, so the achieved BER is not uniform for all of the channels, which is reflected by the number of errors occurring in the received WATM cells. As an example, in Figure 1 we present histograms of the number of transmission errors in a received cell for two of the channels.

For all 13 channels, the obtained BER is equal to $2.8 \times 10^{-3}$, ranging from $1.5 \times 10^{-3}$ to $3.9 \times 10^{-3}$, and the histogram presenting the distribution of errors in the received WATM cells is presented in Figure 2.
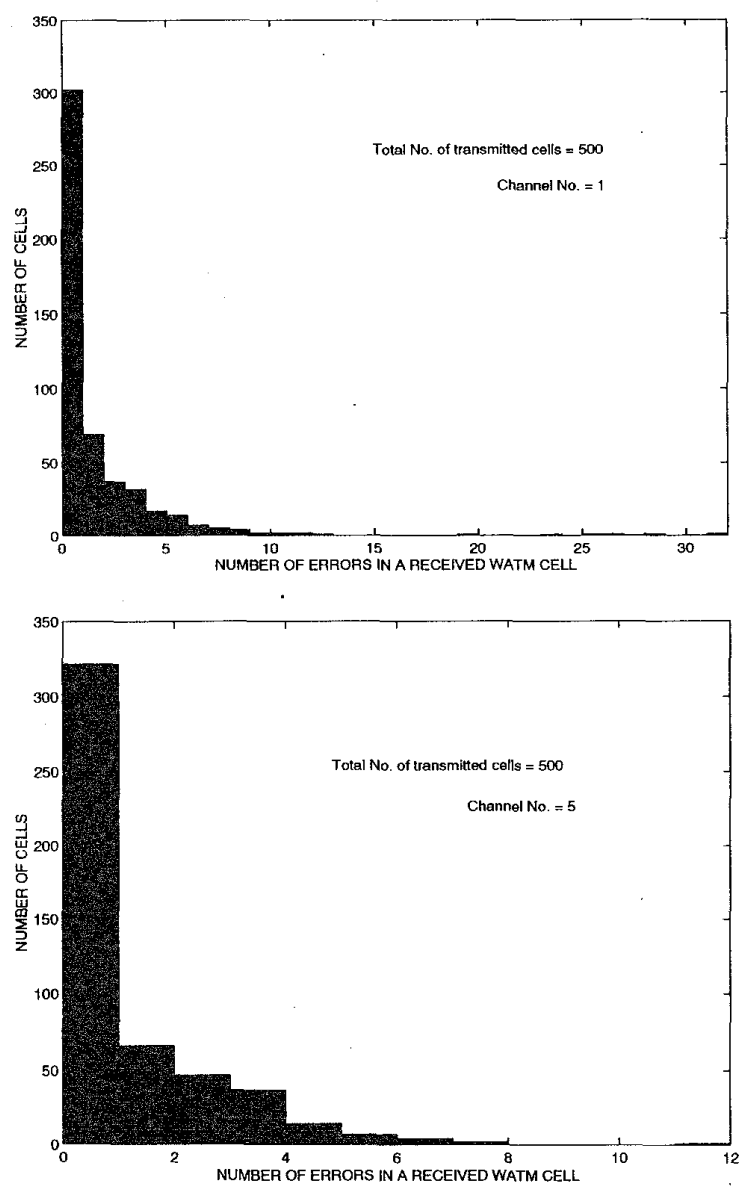

Figure 1: Histograms of the numbers of transmission errors in a WATM cell.

\section{CONCLUSIONS}

In this paper, we presented the simulation results for the 13 channel DS CDMA WATM LAN utilising optimized complex spreading signatures based on WalshRademacher functions. The results are very promising, and indicate that with the application of a hybrid ARQ scheme, capable of correcting 10 errors [7], the number of WATM cells which would require retransmission is in the order of only $1-2 \%$. Further research investigating the behaviour of the system without the perfect power control and incorporating the realistic indoor channel model is required.

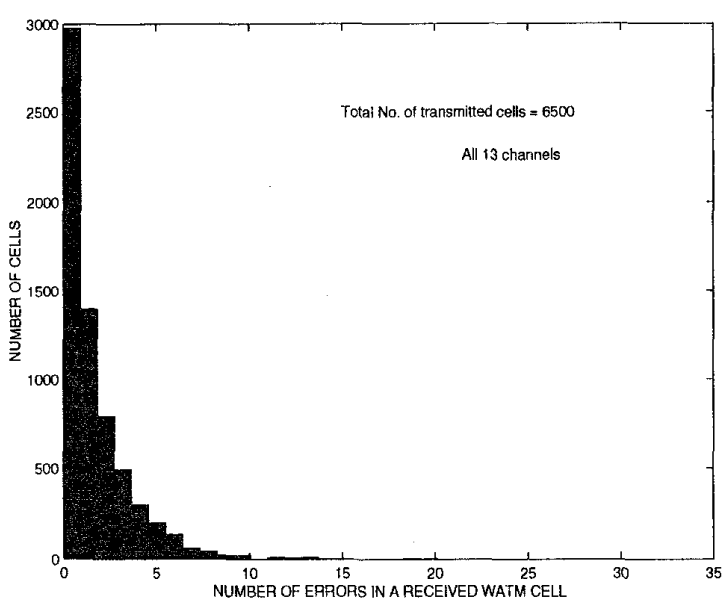

Figure 2: Histograms of the numbers of transmission errors in a received WATM cell for all 13 channels.

\section{REFERENCES}

[1] T.Phipps, et.al.; "Wireless ATM Physical Layer Requirements", ATM Forum, Doc. 96-1004, Aug.1996.

[2] J.G.Proakis; "Digital Communications", 3rd ed., McGraw-Hill, New York, 1995.

[3] B.Wysocki, T.Wysocki; "A Method to Partially Suppress ISI and MAI for DS SS CDMA Wireless Networks", ICC'97, Vol.2, pp.899-903, Montreal 1997.

[4] P. Fan, M.Darnell; "Sequence Design for Communications Applications", John Wiley \& Sons , New York, 1996.

[5] J.A.Nelder, R.Mead; "A Simplex Method for Function Minimization", Computer Journal, Vol.7, pp.308-313.

[6] Anonymous; "MATLAB - Reference Guide", The Math Works Inc., August 1992.

[7] S.G.Fischer, T.A.Wysocki, and H.-J.Zepernick; "MAC Protocol for a CDMA Based Wireless ATM LAN", ICC'97, Vol.3, pp.1202-1206, Montreal 1997. 